\title{
Extratos etanólicos de manga como antioxidantes na alimentação de poedeiras
}

\author{
Ednardo Rodrigues Freitas(1), Ângela da Silva Borges ${ }^{(2)}$, Maria Teresa Salles Trevisan ${ }^{(3)}$, André Luís da Cunha ${ }^{(2)}$, \\ Nádia de Melo Braz ${ }^{(1)}$, Pedro Henrique Watanabe ${ }^{(1)}$ e Germano Augusto Jerônimo do Nascimento ${ }^{(1)}$

\begin{abstract}
(1)Universidade Federal do Ceará (UFC), Centro de Ciências Agrárias, Departamento de Zootecnia, Campus do Pici, Avenida Mister Hull, no 2.977, Caixa Postal 12.168, CEP 60021-970 Fortaleza, CE. E-mail: ednardo@ufc.br, nadia_zootec@yahoo.com.br, pedrowatanabe@ufc.br, germanoaugusto@ufc.br (2)Universidade Federal do Maranhão, Departamento de Engenharia de Alimentos, Campus Universitário de Imperatriz, Rua Urbano Santos, s/no, CEP 65900-410 Imperatriz, MA. E-mail: angelasborges@yahoo.com.br, andreluiscunha@yahoo.com.br
\end{abstract} \\ (3)UFC, Departamento de Química Orgânica e Inorgânica. E-mail: trevisan@ufc.br
}

Resumo - O objetivo deste trabalho foi avaliar o efeito de extratos etanólicos do caroço e da casca de manga, sobre o desempenho de poedeiras e sobre a qualidade e estabilidade lipídica dos ovos. Um total de 180 poedeiras comerciais Hisex White foi distribuído ao acaso em seis tratamentos, com cinco repetições de seis aves. Os tratamentos consistiram de: ração sem adição de antioxidante; ração com 200 ppm do antioxidante butilato de hidroxitolueno (BHT); ração com 200 ou 400 ppm de extrato da casca de manga (Ecas); ração com 200 ou 400 ppm de extrato de caroço de manga (Ecar). Foram avaliados: o consumo de ração, a produção de ovos, o peso do ovo, a massa de ovo produzida (grama por ave por dia), a conversão alimentar e características de qualidade dos ovos. A oxidação lipídica da gema durante o armazenamento foi determinada pela quantificação das substâncias reativas ao ácido tiobarbitúrico. As aves alimentadas com a ração sem adição de antioxidantes produziram ovos com os piores valores de unidade Haugh e maior oxidação lipídica da gema. Os teores de 400 ppm de Ecas e 200 ou 400 ppm de Ecar foram efetivos na prevenção de danos oxidativos aos ovos durante o armazenamento e podem ser utilizados na alimentação das poedeiras como substituto ao antioxidante sintético.

Termos para indexação: Gallus gallus, Mangifera indica, antioxidante natural, mangiferina, oxidação lipídica, qualidade dos ovos.

\section{Ethanol extracts of mango as antioxidants for laying hens}

\begin{abstract}
The objective of this work was to evaluate the effects of ethanol extracts of mango seeds and peel on laying hen performance, egg quality, and egg lipid stability. A total of 180 Hisex White hens were randomly distributed in six treatments, with five replicates of six birds. The treatments consisted of: ration without antioxidant; ration with $200 \mathrm{ppm}$ butylated hydroxytoluene (BHT); ration with 200 or $400 \mathrm{ppm}$ of mango peel extract (Ecas); and ration with 200 or 400 ppm of mango seed extract (Ecar). Feed intake, egg production, egg weight, produced egg mass (gram by bird per day), feed conversion, and egg quality were evaluated. Yolk lipid oxidation during storage was determined by quantification of thiobarbituric acid-reactive substances. Birds fed diet without antioxidants produced eggs with the lowest Haugh unit values and the highest lipid oxidation of yolk. Ecas at $400 \mathrm{ppm}$ and Ecar at 200 or $400 \mathrm{ppm}$ were effective to prevent oxidative damage of eggs during storage and may be used in the diet of laying hens as a replacement for synthetic antioxidant.
\end{abstract}

Index terms: Gallus gallus, Mangifera indica, natural antioxidant, magiferin, lipid oxidation, egg quality.

\section{Introdução}

O ovo é uma excelente fonte de ácidos graxos essenciais que, como em qualquer alimento, estão sujeitos à oxidação lipídica, principalmente durante a estocagem (Hayat et al., 2010). Embora o processo oxidativo em ovos não seja visto como um problema, o aumento da produção de ovos enriquecidos com ácidos graxos poli-insaturados pode aumentar a suscetibilidade à peroxidação. Portanto, há necessidade de estudos que visem ao retardamento desse processo (Radwan et al., 2008; Hayat et al., 2010).

A oxidação lipídica é considerada a deterioração mais importante que ocorre nos alimentos, pois afeta sua qualidade - principalmente o aroma, o sabor e o valor nutricional - e resulta na produção de compostos tóxicos (Amensour et al., 2010). Assim, tendo em vista a proteção dos ovos contra os efeitos da oxidação lipídica, algumas pesquisas têm sido realizadas com antioxidantes sintéticos e naturais na alimentação das 
aves (Hayat et al., 2010). Os antioxidantes sintéticos comumente usados nas rações são o butilato de hidroxianisol (BHA) e o butilato de hidroxitolueno (BHT). Porém, relatos de uma possível ação carcinogênica dessas substâncias têm levado os consumidores a rejeitar produtos que as contenham (Luna et al., 2010). Assim, é importante que se busque substitutos aos antioxidantes sintéticos.

Extratos e óleos essenciais de plantas têm sido relatados como importantes fontes de antioxidantes naturais (Hayat et al., 2010; Luna et al., 2010; Botsoglou et al., 2012). Algumas fontes vegetais ricas em compostos com atividade antioxidante têm sido utilizadas na alimentação de poedeiras, na busca pela melhoria do desempenho das aves e da qualidade dos ovos (Botsoglou et al., 2012). Radwan et al. (2008) constataram que a inclusão na ração de $1 \%$ de ervas - orégano (Origanum sp.), alecrim (Rosmarinus officinalis L) ou tomilho (Thymus vulgaris) - ou 0,5\% de açafrão (Cúrcuma Longa L.) pode melhorar o desempenho produtivo das galinhas, beneficiar a estabilidade oxidativa dos ovos e reduzir a oxidação dos lipídios da gema durante o armazenamento. Zhao et al. (2011) constataram que o gengibre em pó, adicionado à ração de poedeiras, aumentou a massa de ovos produzidos e a estabilidade lipídica da ração e dos ovos durante o armazenamento. Özeku et al. (2011) relataram melhoria significativa da altura do albúmen e valores de unidade Haugh, em ovos de poedeiras alimentadas com mistura de óleos essenciais de orégano, louro, sálvia, murta, erva doce e citrus. Botsoglou et al. (2012) verificaram que o uso das folhas de oliveira (Olea europea L.), na ração de poedeiras voltadas à ovos enriquecidos com ácidos graxos ômega-3, promoveu maior estabilidade lipídica das gemas dos ovos.

A manga (Mangifera indica L.) é uma fruta tropical abundante no Brasil e, por se tratar de fruta sazonal, a maior parte da produção é processada. Em todo o mundo, essa atividade produz grande volume de resíduos, que não são utilizados para nenhum fim comercial e são descartados, tornando-se fonte de poluição (Huber et al., 2012; Dorta et al., 2013). Entretanto, a casca e o caroço da manga são boas fontes de antioxidantes naturais (Huber et al., 2012), pois contêm diferentes componentes com essa ação, entre as quais a provitamina $\mathrm{A}$, na forma de $\beta$-caroteno, e as vitaminas C e E (Puravankara et al., 2000; Abdalla et al., 2007; Ajila et al., 2007), além do composto fenol glicosilxantona, na forma de mangiferina, que tem atividade antioxidante comprovada (Puravankara et al., 2000; Bernardini et al., 2005; Abdalla et al., 2007; Barreto et al., 2008).

Freitas et al. (2012) relatam que a adição de 200 ou 400 ppm dos extratos etanólicos obtidos da casca ou do caroço de manga não influencia o desempenho de frangos de corte, mas podem ser mais efetivas em retardar a oxidação lipídica da carne de frangos do que o uso do antioxidante sintético BHT. No entanto, não há informação a respeito dos efeitos antioxidativos dos extratos etanólicos de manga sobre a produção e a qualidade de ovos de aves em postura.

O objetivo deste trabalho foi avaliar os efeitos de extratos etanólicos do caroço e da casca de manga, sobre o desempenho de poedeiras e sobre a qualidade e estabilidade lipídica dos ovos.

\section{Material e Métodos}

Foram utilizadas 180 poedeiras comerciais Hisex White, com 40 semanas de idade, alojadas em gaiolas de arame galvanizado $(25 \times 40 \times 30 \mathrm{~cm})$, à densidade de duas aves por gaiola. As aves foram distribuídas em delineamento inteiramente casualizado, com seis tratamentos e cinco repetições de seis aves por tratamento. Os tratamentos consistiram de: ração sem adição de antioxidante (controle); ração com adição de 200 ppm de BHT; ração com 200 ppm de extrato de casca de manga (Ecas); ração com 400 ppm de Ecas; ração com 200 ppm de extrato de caroço de manga (Ecar); e ração com 400 ppm de Ecar.

As rações experimentais (Tabela 1) utilizadas eram isonutrientes, de acordo com as exigências nutricionais das aves, pelas recomendações do manual de manejo da linhagem (Manual de manejo Hisex White, 2008), e conforme os valores de composição dos alimentos propostos por Rostagno (2005).

Para a preparação dos extratos naturais, cascas e caroços de frutos maduros de mangas das variedades Coité e Jasmim foram adquiridos em uma indústria de processamento de polpas de frutas e submetidos à secagem em estufa, a $50^{\circ} \mathrm{C}$ por 24 horas, para as cascas, e 48 horas, para os caroços. Em seguida, cada material foi triturado e submetido à extração exaustiva a frio, com os solventes orgânicos hexano e etanol (Barreto et al., 2008). Inicialmente, os materiais 
(5 $\mathrm{kg}$ de cascas e $3 \mathrm{~kg}$ de caroços) foram colocados separadamente em recipientes de vidro, onde permaneceram imersos em $10 \mathrm{~L}$ de hexano, durante sete dias, à temperatura ambiente $\left(25^{\circ} \mathrm{C}\right)$. Após a extração, o material foi filtrado, e o extrato foi concentrado em rotaevaporador Waterbath B-480 (Büchi Labortechnik, $\mathrm{AG}$, Flawil, Suíça) a $50^{\circ} \mathrm{C}$, à rotação de $60 \mathrm{rpm}$ e à pressão reduzida, para obtenção do extrato hexânico e do solvente recuperado, o qual foi utilizado para reextração por mais duas vezes, conforme as condições da extração inicial. Os extratos hexânicos obtidos das três extrações foram descartados, e os resíduos dos materiais foram utilizados para extração etanólica, nas mesmas condições já descritas quanto à extração hexânica. Foram utilizados nas rações apenas os extratos etanólicos a partir das cascas e caroços. Como os diferentes extratos etanólicos apresentavamse com características de gel, após a pesagem, eles foram diluídos em óleo de soja degomado, para serem misturados em cada ração.

A alimentação das aves e a avaliação da qualidade dos ovos foram realizadas durante o período experimental de 63 dias, dividido em três períodos de 21 dias cada. As aves receberam ração e água à vontade durante todo período experimental, com iluminação de 16 horas diárias de luz. Os comedouros foram abastecidos duas vezes ao dia, no início da manhã e ao final da tarde, e a ração foi movimentada para estimular o consumo. A coleta de ovos foi realizada diariamente, ao final da tarde.

As variáveis de desempenho avaliadas foram: consumo de ração (g/ave/dia), produção de ovos (\%/ave/dia), peso do ovo (g), massa de ovo produzida (g/ave/dia), conversão alimentar ( $\mathrm{kg}$ de ração $\mathrm{kg}^{-1}$ de ovo). A ração oferecida no início de cada período de produção e as sobras, no final, foram pesadas e,

Tabela 1. Composição das rações experimentais.

\begin{tabular}{|c|c|c|c|c|c|c|}
\hline \multirow{2}{*}{$\begin{array}{l}\text { Ingrediente } \\
\left(\mathrm{g} \mathrm{kg}^{-1}\right)\end{array}$} & \multirow[t]{2}{*}{ Controle } & \multirow{2}{*}{$\begin{array}{c}\text { BHT } \\
200 \text { ppm }\end{array}$} & \multicolumn{2}{|c|}{ Extrato etanólico da casca } & \multicolumn{2}{|c|}{ Extrato etanólico do caroço } \\
\hline & & & $200 \mathrm{ppm}$ & $400 \mathrm{ppm}$ & $200 \mathrm{ppm}$ & $400 \mathrm{ppm}$ \\
\hline Milho & 634,6 & 634,6 & 634,6 & 634,6 & 634,6 & 634,6 \\
\hline Farelo de soja & 240,5 & 240,5 & 240,5 & 240,5 & 240,5 & 240,5 \\
\hline Óleo de soja & 10,0 & 10,0 & 10,0 & 10,0 & 10,0 & 10,0 \\
\hline Calcáreo & 90,9 & 90,9 & 90,9 & 90,9 & 90,9 & 90,9 \\
\hline Fosfato bicálcico & 15,7 & 15,7 & 15,7 & 15,7 & 15,7 & 15,7 \\
\hline Metionina & 1,8 & 1,8 & 1,8 & 1,8 & 1,8 & 1,8 \\
\hline Minerais para postura $^{(1)}$ & 0,5 & 0,5 & 0,5 & 0,5 & 0,5 & 0,5 \\
\hline Vitamina para postura ${ }^{(2)}$ & 2,0 & 2,0 & 2,0 & 2,0 & 2,0 & 2,0 \\
\hline Sal & 3,6 & 3,6 & 3,6 & 3,6 & 3,6 & 3,6 \\
\hline Inerte (areia lavada) & 0,4 & 0,2 & 0,2 & 0,0 & 0,2 & 0,0 \\
\hline BHT & 0,0 & 0,2 & 0,0 & 0,0 & 0,0 & 0,0 \\
\hline Extrato de casca & 0,0 & 0,0 & 0,2 & 0,4 & 0,0 & 0,0 \\
\hline Extrato de caroço & 0,0 & 0,0 & 0,0 & 0,0 & 0,2 & 0,4 \\
\hline \multirow[t]{2}{*}{ Total } & $1.000,0$ & $1.000,0$ & $1.000,0$ & $1.000,0$ & $1.000,0$ & $1.000,0$ \\
\hline & \multicolumn{6}{|c|}{ Composição nutricional e energética } \\
\hline Energia metabólica $\left(\mathrm{kcal} \mathrm{kg}^{-1}\right)$ & 2.800 & 2.800 & 2.800 & 2.8000 & 2.800 & 2.800 \\
\hline Proteína bruta $\left(\mathrm{g} \mathrm{kg}^{-1}\right)$ & 165,0 & 165,0 & 165,0 & 165,0 & 165,0 & 165,0 \\
\hline Ácido linoleico $\left(\mathrm{g} \mathrm{kg}^{-1}\right)$ & 19,3 & 19,3 & 19,3 & 19,3 & 19,3 & 19,3 \\
\hline Cálcio $\left(\mathrm{g} \mathrm{kg}^{-1}\right)$ & 39,0 & 39,0 & 39,0 & 39,0 & 39,0 & 39,0 \\
\hline Fósforo disponível $\left(\mathrm{g} \mathrm{kg}^{-1}\right)$ & 4,0 & 4,0 & 4,0 & 4,0 & 4,0 & 4,0 \\
\hline Sódio $\left(\mathrm{g} \mathrm{kg}^{-1}\right)$ & 1,8 & 1,8 & 1,8 & 1,8 & 1,8 & 1,8 \\
\hline Cloro $\left(\mathrm{g} \mathrm{kg}^{-1}\right)$ & 2,5 & 2,5 & 2,5 & 2,5 & 2,5 & 2,5 \\
\hline Lisina total $\left(\mathrm{g} \mathrm{kg}^{-1}\right)$ & 8,3 & 8,3 & 8,3 & 8,3 & 8,3 & 8,3 \\
\hline Metionina total $\left(\mathrm{g} \mathrm{kg}^{-1}\right)$ & 4,4 & 4,4 & 4,4 & 4,4 & 4,4 & 4,4 \\
\hline Metionina+ cisteína total $\left(\mathrm{g} \mathrm{kg}^{-1}\right)$ & 7,2 & 7,2 & 7,2 & 7,2 & 7,2 & 7,2 \\
\hline Treonina $\left(\mathrm{g} \mathrm{kg}^{-1}\right)$ & 6,4 & 6,4 & 6,4 & 6,4 & 6,4 & 6,4 \\
\hline Triptofano $\left(\mathrm{g} \mathrm{kg}^{-1}\right)$ & 1,9 & 1,9 & 1,9 & 1,9 & 1,9 & 1,9 \\
\hline
\end{tabular}

${ }^{(1)}$ Minerais (quantidade fornecida por quilograma do produto): cobre, $10 \mathrm{mg}$; zinco, $50 \mathrm{mg}$; ferro, $40 \mathrm{mg}$; manganês, $65 \mathrm{mg}$; iodo, $1 \mathrm{mg}$. (2)Vitamina (quantidade fornecida por quilograma do produto): vitamina A, 7.950 UI; vitamina B1, 1,95 mg; vitamina B12, 13,05 mcg; vitamina B2, 4,95 mg; vitamina B6, 3,30 mg; vitamina D3, $2.200 \mathrm{UI}$; vitamina E, $10,95 \mathrm{mg}$; vitamina K3, 1,80 mg; ácido fólico, 0,81 mg; pantotenato de cálcio, $12,0 \mathrm{mg}$; colina, 0,51 g; niacina, $36,0 \mathrm{mg}$; antioxidante, 10,2 g; coccidiostático, $1,02 \mathrm{~g}$; selênio, $0,15 \mathrm{mg} .{ }^{(3)} \mathrm{BHT}$, butilato de hidroxitolueno. 
por diferença, foi calculado o consumo de ração. A produção de ovos foi registrada diariamente por gaiola e, ao final de cada período, foram calculadas as percentagens de postura. Com base nos dados de consumo de ração e produção de ovos, foi realizado o cálculo de conversão alimentar. Os ovos foram coletados, identificados e armazenados à temperatura ambiente, no último dia de cada semana. Nos dias seguintes, era realizada a pesagem dos ovos de cada repetição, em balança eletrônica de precisão $(0,01 \mathrm{~g})$, para determinar o peso médio dos ovos. A massa de ovos foi calculada pela multiplicação do peso médio dos ovos pela percentagem de postura.

A avaliação da qualidade dos ovos foi realizada uma vez por semana, durante todo o período experimental. Para isso, todos os ovos de cada repetição foram coletados, e três deles foram selecionados, aleatoriamente (tendo-se evitado os ovos quebrados, trincados ou sujos), para serem avaliados. Inicialmente, determinou-se a gravidade específica (GE) dos ovos, conforme Freitas et al. (2004). Após a determinação da GE, os ovos foram quebrados sobre uma superfície de vidro, para a determinação da altura do albúmen, por meio de um micrômetro de profundidade S-6428 (Ames, Waltham, MA, EUA). Os dados da altura do albúmen e do peso dos ovos foram utilizados no cálculo do valor da unidade Haugh (UH), pela equação $\mathrm{UH}=100 \log \left(\mathrm{H}+7,57-1,7 \mathrm{~W}^{0,37}\right)$, em que H é a altura do albúmen (mm), e W é o peso do ovo $(\mathrm{g})$.

Para determinar as proporções $\left(\mathrm{g} \mathrm{kg}^{-1}\right)$ de cada constituinte nos ovos, as gemas foram separadas e pesadas em balança de precisão $(0,01 \mathrm{~g})$, e as cascas foram lavadas, postas para secar à temperatura ambiente por 48 horas, e então pesadas. As proporções de gema e casca foram obtidas pela relação entre o peso de cada porção e o peso do ovo, e a de albúmen foi determinada por diferença: albúmen $=100$ - (gema + casca $)$.

A estabilidade lipídica dos ovos foi avaliada no segundo período experimental, com os ovos produzidos durante cinco dias, dos quais cinco ovos foram selecionados por repetição, com base na ausência de rachaduras, manchas ou sujeiras na casca, para serem submetidos aos períodos de armazenamento de 0,15 , 30,45 e 60 dias, em refrigerador a $4^{\circ} \mathrm{C}$. Após a seleção e identificação, os ovos foram acondicionados em bandejas de papelão, e armazenados. A temperatura de armazenamento foi monitorada diariamente com o uso de um termômetro digital.

A oxidação lipídica foi avaliada por meio da determinação das substâncias reativas ao ácido tiobarbitúrico (Tbars), pelo método de extração acidoaquosa, conforme Kang et al. (2001). Em cada período de armazenamento descrito acima, as gemas dos cinco ovos de cada repetição foram separadas da clara, colocadas em béquer, homogeneizadas e levadas para análise. $\mathrm{O}$ número de substâncias reativas na amostra foi expresso como miligrama de malonaldeído por quilograma de gema.

As análises estatísticas dos dados foram realizadas com o SAS, versão 9.2 (SAS Institute, Cary, NC, EUA), tendo-se adotado 5\% como nível de significância. Os dados de desempenho e qualidade dos ovos foram submetidos à análise de variância (Proc Anova, SAS), em delineamento inteiramente casualizado. Os dados da estabilidade lipídica dos ovos armazenados foram analisados por modelo fatorial, em que foram incluídos os efeitos do tratamento, do tempo de armazenamento e da interação entre o tratamento e o tempo de armazenamento. As médias foram comparadas pelo teste de Student-Newman-Keuls (SNK). Utilizou-se, ainda, a análise de regressão para descrever o efeito do tempo de armazenamento sobre a estabilidade lipídica dos ovos.

\section{Resultados e Discussão}

Não houve diferença significativa entre os tratamentos quanto a: consumo de ração, percentagem de postura, peso médio dos ovos, massa de ovos produzida e conversão alimentar das aves (Tabela 2).

A quantidade de alimento ingerido voluntariamente pelas aves está diretamente relacionada à palatabilidade da ração (Leeson \& Summers, 2001). No entanto, no presente trabalho, não houve diferença na quantidade nesse parâmetro, o que indica que a adição dos extratos não alterou a palatabilidade das rações testadas. Como as rações foram isonutritivas, e não houve diferença no consumo, também não houve diferença significativa no desempenho das aves.

A ausência de influência significativa de fontes naturais de antioxidantes sobre o consumo de ração (Radwan et al., 2008; Zhao et al., 2011; Özeku et al., 2011; Bozkurt et al., 2012) e o desempenho das galinhas (Özeku et al., 2011; Bozkurt et al., 2012) é comum. Entretanto, Radwan et al. (2008) constataram 
que, embora a inclusão de $1 \%$ de orégano, alecrim ou tomilho, ou de $0,5 \%$ de açafrão não tenha influenciado o consumo, as poedeiras tiveram desempenho melhorado com esses tratamentos. Além disso, Zhao et al. (2011) verificaram que a adição de gengibre em pó na ração de poedeiras aumenta a massa do ovo.

Não foi observada diferença significativa entre os tratamentos, quanto à gravidade específica dos ovos e à proporção de gema, albúmen e casca. Entretanto, os valores de unidades Haugh diferiram significativamente (Tabela 3). Os ovos das aves alimentadas com a ração sem adição de antioxidantes (controle) apresentaram os menores valores de unidade Haugh, enquanto os ovos das aves alimentadas com BHT ou com os produtos da manga, não diferiram significativamente entre si. A melhoria da qualidade do albúmen, medida por unidade Haugh, como resposta à ação antioxidante da ração foi relatada por Kucuk et al. (2003), em trabalho sobre a adição conjunta de vitamina $\mathrm{C}$ e $\mathrm{E}$ à ração de poedeiras. A adição dos extratos produziu efeito semelhante aos relatados por Özeku et al. (2011), que apenas constataram aumento na altura do albúmen e nos valores de unidade Haugh, em ovos de poedeiras alimentadas com uma mistura de óleos essenciais. Entretanto, Bozkurt et al. (2012) avaliaram produto semelhante e não observaram efeito sobre a qualidade do albúmen.

A resposta da estabilidade dos lipídeos na gema aos tratamentos, medida pelos valores de Tbars, variou conforme o tempo de armazenamento (Tabela 4), o que foi revelado pela interação significativa $(p<0,0001)$ entre os fatores.

Tabela 2. Desempenho de poedeiras comerciais alimentadas com rações com extratos etanólicos de manga.

\begin{tabular}{lccccc}
\hline Tratamento $^{(1)}$ & \multicolumn{5}{c}{ Variáveis de desempenho } \\
\cline { 2 - 6 } & $\begin{array}{l}\text { Consumo } \\
\text { (g/ave/dia) }\end{array}$ & $\begin{array}{c}\text { Produção } \\
(\% / a v e / d i a)\end{array}$ & $\begin{array}{c}\text { Peso de Massa de ovo } \\
\text { ovo }(\mathrm{g})\end{array}$ & $\begin{array}{c}\text { Conversão } \\
\text { (g/ave/dia) }\end{array}$ & $\begin{array}{c}\text { alimentar } \\
\left(\mathrm{kg} \mathrm{kg}^{-1}\right)\end{array}$ \\
\hline Controle & 95,92 & 95,96 & 59,77 & 57,13 & 1,69 \\
BHT, 200 ppm & 96,32 & 94,83 & 60,66 & 57,09 & 1,69 \\
Ecas, 200 ppm & 97,34 & 93,55 & 60,10 & 55,51 & 1,76 \\
Ecas, 400 ppm & 96,23 & 91,88 & 61,01 & 55,67 & 1,73 \\
Ecar, 200 ppm & 97,57 & 94,39 & 60,20 & 56,55 & 1,72 \\
Ecar, 400 ppm & 97,27 & 94,15 & 60,10 & 56,26 & 1,73 \\
\hline Média & 96,78 & 94,13 & 60,31 & 56,37 & 1,72 \\
CV (\%) & 2,65 & 3,44 & 3,27 & 4,15 & 2,69 \\
\hline
\end{tabular}

(1)BHT, butilato de hidroxitolueno; Ecas, extrato de casca de manga; Ecar, extrato de caroço de manga.
A adição de BHT e dos extratos etanólicos de caroço e de casca de manga reduziu a oxidação lipídica dos ovos (menores valores de Tbars), em todos os tempos de armazenamento. Aos 60 dias, os menores valores de Tbars foram obtidos nos tratamentos com $400 \mathrm{ppm}$ de Ecas, 200 ppm de Ecar e 400 ppm de Ecar, enquanto os ovos das aves que receberam o tratamento-controle apresentaram os maiores valores de Tbars, e os ovos de aves que receberam BHT e 200 ppm de Ecas apresentaram valores intermediários.

Os valores de Tbars aumentaram linearmente com o tempo de armazenamento, para todos os tratamentos (Figura 1). Esse resultado tem sido relatado por outros

Tabela 3. Característica e qualidade de ovos de poedeiras comerciais alimentadas com rações com extratos etanólicos de manga ${ }^{(1)}$.

\begin{tabular}{lccccc}
\hline Tratamento $^{(2)}$ & \multicolumn{5}{c}{ Características dos ovos } \\
\cline { 2 - 6 } & $\begin{array}{c}\text { Gema } \\
\left(\mathrm{g} \mathrm{kg}^{-1}\right)\end{array}$ & $\begin{array}{c}\text { Albúmen } \\
\left(\mathrm{g} \mathrm{kg}^{-1}\right)\end{array}$ & $\begin{array}{c}\text { Casca } \\
\left(\mathrm{g} \mathrm{kg}^{-1}\right)\end{array}$ & $\begin{array}{c}\text { Gravidade } \\
\text { específica } \\
\left(\mathrm{g} \mathrm{cm}^{-3}\right)\end{array}$ & $\begin{array}{c}\text { Unidades } \\
\text { Haugh }\end{array}$ \\
\hline Controle & 264,80 & 638,50 & 99,40 & 1,080 & $87,14 \mathrm{~b}$ \\
BHT, 200 ppm & 256,50 & 648,50 & 98,40 & 1,073 & $90,07 \mathrm{a}$ \\
Ecas, 200 ppm & 256,10 & 648,30 & 97,90 & 1,079 & $90,22 \mathrm{a}$ \\
Ecas, 400 ppm & 254,90 & 647,60 & 98,60 & 1,078 & $90,23 \mathrm{a}$ \\
Ecar, 200 ppm & 259,00 & 644,70 & 96,00 & 1,091 & $90,73 \mathrm{a}$ \\
Ecar, 400 ppm & 251,90 & 650,90 & 96,40 & 1,082 & $92,47 \mathrm{a}$ \\
\hline Média & 257,20 & 646,42 & 97,78 & 1,081 & 90,14 \\
CV (\%) & 3,00 & 1,34 & 2,16 & 0,2426 & 1,86 \\
\hline
\end{tabular}

${ }^{(1)}$ Médias seguidas por letras iguais não diferem, pelo teste SNK, a $5 \%$ de probabilidade. ${ }^{(2)}$ BHT, butilato de hidroxitolueno; Ecas, extrato de casca de manga; Ecar, extrato de caroço de manga.

Tabela 4. Efeito do armazenamento sobre os valores de Tbars ( $\mathrm{mg} \mathrm{kg}^{-1}$ de malonaldeído), em gemas de ovos de poedeiras alimentadas com rações com extratos etanólicos de manga ${ }^{(1)}$.

\begin{tabular}{lcccccc}
\hline Tratamento $^{(2)}$ & \multicolumn{5}{c}{ Tempo de armazenamento (dias) } & Média \\
\cline { 2 - 7 } & 0 & 15 & 30 & 45 & 60 & \\
\hline Controle & $0,86 \mathrm{a}$ & $1,19 \mathrm{a}$ & $1,24 \mathrm{a}$ & $1,41 \mathrm{a}$ & $1,53 \mathrm{a}$ & 1,25 \\
BHT, 200 ppm & $0,62 \mathrm{~b}$ & $0,63 \mathrm{~b}$ & $0,71 \mathrm{~b}$ & $0,90 \mathrm{~b}$ & $0,94 \mathrm{~b}$ & 0,76 \\
Ecas, 200 ppm & $0,60 \mathrm{~b}$ & $0,62 \mathrm{~b}$ & $0,79 \mathrm{~b}$ & $0,91 \mathrm{~b}$ & $0,93 \mathrm{~b}$ & 0,77 \\
Ecas, 400 ppm & $0,64 \mathrm{~b}$ & $0,65 \mathrm{~b}$ & $0,76 \mathrm{~b}$ & $0,79 \mathrm{~b}$ & $0,82 \mathrm{c}$ & 0,73 \\
Ecar, 200 ppm & $0,69 \mathrm{~b}$ & $0,70 \mathrm{~b}$ & $0,79 \mathrm{~b}$ & $0,83 \mathrm{~b}$ & $0,76 \mathrm{c}$ & 0,75 \\
Ecar, 400 ppm & $0,63 \mathrm{~b}$ & $0,68 \mathrm{~b}$ & $0,73 \mathrm{~b}$ & $0,79 \mathrm{~b}$ & $0,82 \mathrm{c}$ & 0,73 \\
\hline Média & 0,67 & 0,74 & 0,84 & 0,94 & 0,97 & \\
\hline
\end{tabular}

${ }^{(1)}$ Médias seguidas por letras iguais, nas colunas, não diferem pelo teste SNK, a $5 \%$ de probabilidade. ${ }^{(2)} \mathrm{BHT}$, butilato de hidroxitolueno; Ecas, extrato de casca de manga; Ecar, extrato de caroço de manga. CV, coeficiente de variação $=11,33$. 
pesquisadores (Tserveni-Gousi et al., 2006; Radwan et al., 2008; Shahryar et al., 2010; Zhao et al., 2011). Segundo Shahryar et al. (2010), o nível de Tbars nos ovos, ao final do período de armazenamento, está diretamente relacionado à composição lipídica e à transferência do antioxidante para os ovos. Assim, ovos enriquecidos com ácidos graxos insaturados são mais suscetíveis à oxidação lipídica e, consequentemente, apresentarão maiores valores de Tbars, se o antioxidante adicionado à ração não for transferido para os ovos em quantidade suficiente.

Radwan et al. (2008) avaliaram os valores de Tbars em ovos frescos, antes do armazenamento, e relataram que a concentração de substâncias reativas ao ácido tiobarbitúrico, em ovos recém-postos, pode ser decorrente da ingestão dessas substâncias na ração e da posterior transferência para a gema ou, ainda, resultante da produção endógena das poedeiras. Assim, a adição de antioxidantes à ração reduz os valores de Tbars.

A atividade antioxidante dos extratos de casca e de caroço de manga tem sido associada à ação de diferentes substâncias. Alguns estudos indicaram que a manga é uma boa fonte de antioxidantes naturais, com destaque para mangiferina, um composto de fenol glicosilxantona (Puravankara et al., 2000; Abdalla et al., 2007). A mangiferina tem atividade antioxidante comprovada (Barreto et al., 2008), e a proporção deste composto varia conforme a cultivar e as partes do fruto (Bernardini et al., 2005; Barreto et al., 2008).

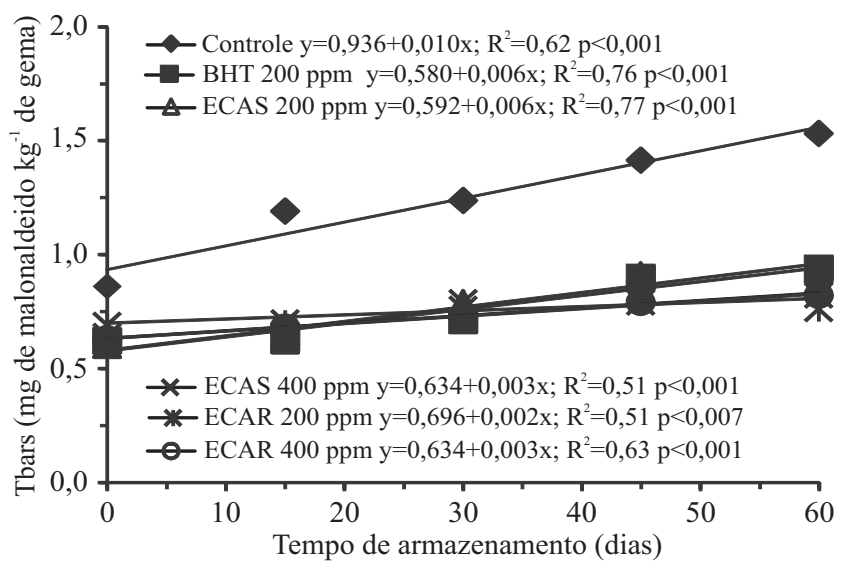

Figura 1. Valores de Tbars em gemas de ovos de poedeiras alimentadas com rações contendo extratos etanólicos de manga, ao longo do tempo de armazenamento. BHT, butilato de hidroxitolueno; Ecas, extrato de casca de manga; Ecar, extrato de caroço de manga.
Embora a adição de ambos os extratos etanólicos à ração tenha diminuído os valores de Tbars nos ovos, os extratos da casca de manga não apresentaram a mesma eficiência que os do caroço, após 60 dias de armazenamento (Tabela 4). Com esse tempo de armazenamento, os ovos das aves alimentadas com 200 ppm de Ecas apresentaram valor de Tbars significativamente maior que os ovos das aves alimentadas com 200 ppm de Ecar. Essa diferença na ação antioxidante, possivelmente, está associada a diferenças na composição dos extratos. Schieber et al. (2003) e Barreto et al. (2008) mostraram que há diferença entre casca e caroço de manga, quanto ao tipo e ao conteúdo de compostos fenólicos presentes. Ribeiro et al. (2008) mostraram que o teor de compostos fenólicos presentes no caroço de manga da variedade Ubá foi superior ao da casca ( 8,25 vs. 5,72\%). Além disso, de acordo com Abdalla et al. (2007), o caroço de manga é uma excelente fonte de tocoferóis, que também têm propriedades antioxidantes e apresentam, ainda, fator de inibição potencial de catalisadores oxidativos, como a tirosinase (Maisuthisakul \& Gordon, 2009).

A diferença entre a ação antioxidante dos extratos etanólicos de casca e de caroço, verificada na presente pesquisa, é semelhante à relatada por Freitas et al. (2012), que verificaram que a adição 200 ppm de Ecas foi menos eficiente do que a de $200 \mathrm{ppm}$ de Ecar, em prevenir a oxidação lipídica da carne de frango armazenada por 15 dias. Os autores relataram a dose de 400 ppm de Ecas como necessária à obtenção de efeito semelhante ao obtido com 200 ou 400 ppm de Ecar.

Os efeitos dos extratos de manga sobre a oxidação lipídica da gema corroboram uma das respostas, frequentemente relatadas na literatura (Radwan et al., 2008; Zhao et al., 2011), de que a adição de antioxidantes naturais beneficia a qualidade dos ovos, principalmente, na proteção contra a oxidação dos lipídeos da gema.

\section{Conclusões}

1. A adição de antioxidante sintético ou dos extratos etanólicos de manga não afeta o desempenho de poedeiras e melhora a qualidade do albúmen e a estabilidade lipídica dos ovos.

2. O extrato etanólico de caroço de manga é mais eficiente do que o de casca, em retardar a oxidação lipídica de ovos armazenados por até 60 dias.

Pesq. agropec. bras., Brasília, v.48, n.7, p.714-721, jul. 2013 DOI: 10.1590/S0100-204X2013000700003 


\section{Referências}

ABDALLA, A.E.M.; DARWISH, S.M.; AYAD, E.H.E.; EL-HAMAHMY, R.M. Egyptian mango by-product 2: antioxidant and antimicrobial activities of extract and oil from mango seed kernel. Food Chemistry, v.103, p.1141-1152, 2007. DOI: 10.1016/j.foodchem.2006.10.026.

AJILA, C.M.; NAIDU, K.A.; BHAT, S.G.; PRASADA RAO, U.J.S. Bioactive compounds and antioxidant potential of mango peel extract. Food Chemistry, v.105, p.982-988, 2007. DOI: 10.1016/j.foodchem.2007.04.052.

AMENSOUR, M.; SENDRA, E.; ABRINI, J.; PÉREZ-ALVAREZ, J.A.; FERNÁNDEZ-LÓPEZ, J. Antioxidant activity and total phenolic compounds of myrtle extracts. CyTA - Journal of Food, v.8, p 95-101, 2010. DOI: 10.1080/19476330903161335.

BARRETO, J.C.; TREVISAN, M.T.S.; HULL, W.E.; ERBEN, G.; BRITO, E.S. de; PFUNDSTEIN, B.; WURTELE, G.; SPIEGELHALDER, B.; OWEN, R.W. Characterization and quantitation of polyphenolic compounds in bark, kernel, leaves, and peel of mango (Mangifera indica L.). Journal of Agricultural and Food Chemistry, v.56, p.5599-5610, 2008. DOI: 10.1021/ jf800738r.

BERNARDINI, N.; FEZER, R.; CONRAD, J.; BEIFUSS, U.; CARLE, R.; SCHIEBER, A. Screening of mango (Mangifera indica L.) cultivars for their contents of flavonol O- and xanthone C-glycosides, anthocyanins, and pectin. Journal of Agricultural and Food Chemistry, v.53, p.1563-1570, 2005. DOI: 10.1021/ jf0484069.

BOTSOGLOU, E.; GOVARIS, A.; FLETOURIS, D.; ILIADIS, S. Olive leaves (Olea europea L.) and $\alpha$-tocopheryl acetate as feed antioxidants for improving the oxidative stability of $\alpha$-linolenic acid-enriched eggs. Journal of Animal Physiology and Animal Nutrition, v.97, p.740-753, 2012. DOI: 10.1111/j.1439-0396.201 2.01316.x.

BOZKURT, M.; KÜÇÜKYILMAZ, K.; ÇATLI, A.U.; ÇINAR, M.; BINTAŞ, E.; ÇÖVEN, F. Performance, egg quality, and immune response of laying hens fed diets supplemented with mannan-oligosaccharide or an essential oil mixture under moderate and hot environmental conditions. Poultry Science, v.91, p.1379-1386, 2012. DOI: 10.3382/ps.2011-02023.

DORTA, E.; LOBO, M.G.; GONZÁLEZ, M. Improving the efficiency of antioxidant extraction from mango peel by using microwave-assisted extraction. Plant Foods for Human Nutrition, v.68, p.190-199, 2013. DOI: 10.1007/s11130-013-0350-4.

FREITAS, E.R.; BORGES, A. da S.; TREVISAN, M.T.S.; WATANABE, P.H.; CUNHA, A.L. da; PEREIRA, A.L.F.; ABREU, V.K.; NASCIMENTO, G.A.J. do. Extratos etanólicos da manga como antioxidantes para frangos de corte. Pesquisa Agropecuária Brasileira, v.47, p.1025-1030, 2012. DOI: 10.1590/ S0100-204X2012000800001.

FREITAS, E.R.; SAKOMURA, N.K.; GONZALES, M.M.; BARBOSA, N.A.A. Comparação de métodos de determinação da gravidade específica de ovos de poedeiras comerciais. Pesquisa Agropecuária Brasileira, v.39, p.509-512, 2004. DOI: 10.1590/ S0100-204X2004000500014.
HAYAT, Z.; CHERIAN, G.; PASHA,T.N.; KHATTAK, F.M.; JABBAR, M.A. Oxidative stability and lipid components of eggs from flax-fed hens: effect of dietary antioxidants and storage. Poultry Science, v.89, p.1285-1292, 2010. DOI: 10.3382/ ps.2009-00256.

HUBER, K.; QUEIROZ, J.H. de; MOREIRA, A.V.B.; RIBEIRO, S.M.R. Caracterização química do resíduo agroindustrial da manga Ubá (Mangifera indica L.): uma perspectiva para a obtenção de antioxidantes naturais. Revista Brasileira de Tecnologia Agroindustrial, v.6, p.640-654, 2012. DOI: 10.3895/ S1981-36862012000100003.

KANG, K.R.; CHERIAN, G.; SIM, J.S. Dietary palm oil alters the lipid stability of polyunsaturated fatty acid-modified poultry products. Poultry Science, v.80, p.228-234, 2001.

KUCUK, O.; SAHIN, N.; SAHIN, K.; GURSU, M.F.; GULCU, F.; OZCELIK, M.; ISSI, M. Egg production, egg quality, and lipid peroxidation status in laying hens maintained at a low ambient temperature $\left(6^{\circ} \mathrm{C}\right)$ and fed a vitamin $\mathrm{C}$ and vitamin E-supplemented diet. Veterinární Medicína, v.48, p.33-40, 2003.

LEESON, S.; SUMMERS, J.D. Nutrition of the chicken. $4^{\text {th }}$ ed. Ontario: University Books, 2001. 591p.

LUNA, A.; LÁBAQUE, M.C.; ZYGADLO, J.A.; MARIN, R.H. Effects of thymol and carvacrol feed supplementation on lipid oxidation in broiler meat. Poultry Science, v.89, p.366-370, 2010. DOI: $10.3382 /$ ps.2009-00130.

MAISUTHISAKUL, P.; GORDON, M.H. Antioxidant and tyrosinase inhibitory activity of mango seed kernel by product. Food Chemistry, v.117, p.332-341, 2009. DOI: 10.1016/j. foodchem.2009.04.010.

MANUAL de manejo Hisex White. Cascavel: Interaves, 2008. 55p.

ÖZEKU, K.; WELLMANN, K.T.; ERTEKIN, B.; TARIM, B. Effects of dietary herbal essential oil mixture and organic acid preparation on laying traits, gastrointestinal tract characteristics, blood parameters and immune response of laying in a hot summer season. Journal of Animal and Feed Sciences, v.20, p.575-586, 2011.

PURAVANKARA, D.; BOHGRA, V.; SHARMA, R.S. Effect of antioxidant principles isolated from mango (Mangifera indica L.) seed kernels on oxidative stability of buffalo ghee (butter-fat). Journal of the Science of Food and Agriculture, v.80, p.522-526, 2000. DOI: $10.1002 /(\mathrm{SICI}) 1097-0010(200003) 80: 4<522::$ AID-JS FA560>3.0.CO;2-R.

RADWAN, L.N.; HASSAN, R.A.; QOTA, E.M.; FAYEK H.M. Effect of natural antioxidant on oxidative stability of eggs and productive and reproductive performance of laying hens. International Journal of Poultry Science, v.7, p.134-150, 2008. DOI: 10.3923/ijps.2008.134.150.

RIBEIRO, S.M.R.; BARBOSA, L.C.A.; QUEIROZ, J.H.; KNODLER, M.; SCHIEBER, A. Phenolic compounds and antioxidant capacity of Brazilian mango (Mangifera indica L.) varieties. Food Chemistry, v.110, p.620-626, 2008. DOI: 10.1016/j.foodchem.2008.02.067.

ROSTAGNO, H.S. (Ed.). Tabelas brasileiras para aves e suínos: composição de alimentos e exigências nutricionais. 2.ed. Viçosa: UFV/DZO, 2005. 186p. 
SCHIEBER, A.; BERARDINI, N.; CARLE, R. Identification of flavonol and xanthone glycosides from mango (Mangifera indica L. cv. 'Tommy Atkins') peels by high-performance liquid chromatography-electrospray ionization mass spectrometry. Journal of Agricultural and Food Chemistry, v.51, p.5006-5011, 2003. DOI: $10.1021 /$ jf030218f.

SHAHRYAR, H.A.; SALAMATDOUST, R.; CHEKANI-AZAR, S.; AHADI, F.; VAHDATPOOR, T. Lipid oxidation in fresh and stored eggs enriched with dietary ômega 3 and ômega 6 polyunsaturated fatty acids and vitamin $\mathrm{E}$ and A dosages. African Journal of Biotechnology, v.9, p.1827-1832, 2010.
TSERVENI-GOUSI, A.; YANNAKOPOULOS, A.; BOTSOGLOU, N.; CHRISTAKI, E.; FLOROU-PANERI, P.; YANNAKAKIS, E. Sensory evaluation and oxidative stability of n-3 fatty acid enriched eggs in Greece. Archiv für Geflügelkunde, v.70, p.228-231, 2006.

ZHAO, X.; YANG, Z.B.; YANG, W.R.; WANG, Y.; JIANG, S.Z.; ZHANG, G.G. Effects of ginger root (Zingiber officinale) on laying performance and antioxidant status of laying hens and on dietary oxidation stability. Poultry Science, v.90, p.1720-1727, 2011. DOI: $10.3382 /$ ps.2010-01280.

Recebido em 27 de dezembro de 2012 e aprovado em 28 de agosto de 2013 